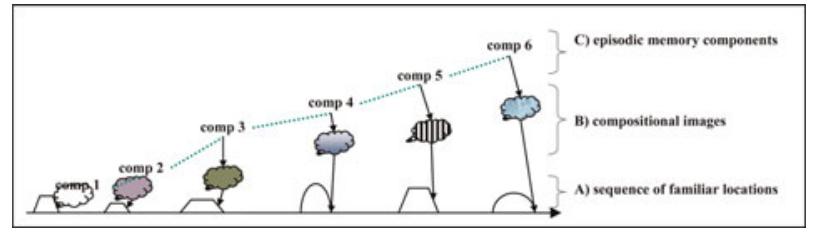

Figure 1 (Nielsen). Encoding of an episodic memory with the classical method of loci technique. The individual draws upon (A) a familiar sequence of locations, such as a building or path with a known order. To each unique location, the individual associates (B) a distinctive compositional image that substitutes for (C) a component of the to-be-remembered episode. To recall the memory, the individual mentally "revisits" the locations and "retrieves" the image/component "placed" there. Because the location sequence is familiar, images linked to it can be accessed in any order. Distinctiveness of the compositional images derives from, for example, their visual vividness, bizarreness, or emotion. Dream imagery only rarely depicts familiar, coherently ordered sequences of locations but may at times fulfill the requirement of distinctiveness (see the text).

Invention of the ancient art of memory (AAOM) is attributed to Greek poet Simonides of Ceos (556-468 BCE), who developed the method of loci (MoL; Fig. 1) after purportedly identifying the remains of comrades crushed in a temple collapse solely from his memory of where they were sitting before he fortuitously left the building. The MoL became common in Greek oral traditions and remains among the most effective mnemonic techniques (Massen et al. 2009; Verhaeghen \& Kliegl 2000). The MoL facilitates memory for both abstract and concrete material (Wang \& Thomas 2000), is especially effective with serially ordered material (e.g., episodic memories) and is most effective when the imagined pathways used are self-generated versus other-generated (Bellezza \& Reddy 1978). It is a favored technique of mnemonists, as shown in a study of highly trained subjects, $90 \%$ of whom spontaneously used the MoL to complete study tasks (Maguire et al. 2003). Some findings even suggest that the MoL is effective without using bizarre or atypical composite imagery; the latter have no effect on memory if the imagery employed is sufficiently concrete, lively, and emotional (Persensky \& Senter 1970; Senter \& Hoffman 1976).

Given the centrality of the MoL technique to memory enhancement, a critical question for Llewellyn's hypothesis is: Does dreaming portray coherently ordered, familiar locations like those required for the MoL?

Evidence supports the view that it does not, that dream locations are neither familiar nor coherently ordered. First, subjects rate dream locations as familiar only infrequently. In one study (119 dreams, 331 locations), they indicated that only $35.5 \%$ of dream locations were either exact or slightly modified replications of previously experienced locations (Dorus et al. 1971). This value is lower than for characters $(51.7 \%)$ or activities/interactions $(39.1 \%)$ but parallels the $33.7 \%$ of dream settings that subjects in a normative study $(N=200)$ described as familiar (Hall \& van de Castle 1966). The latter value is an overestimate because familiarity scoring included famous settings that subjects may never have visited. Accordingly, another study estimates that familiar settings are the least frequent episodic memory element (17\%) that subjects link to their dreams; less frequent even than themes, emotions, characters, or actions (Fosse et al. 2003).

Second, a lack of location ordering is suggested by the finding that dream locations are clearly the most frequent temporally discontinuous element (12.8\%); they suddenly and unexpectedly appear, disappear, or transform (Revonsuo \& Salmivalli 1995). Other elements, such as persons, objects, or actions, are discontinuous only $1.5 \%-4.8 \%$ of the time. A second study (Rittenhouse et al. 1994) found discontinuities of location (10\%) to be second only to discontinuities of plot (14\%). Beyond such apparent discontinuity, however, successive dream locations may not be linked in any coherent sense (see example below). A dream protagonist might traverse several locations that are not logically connected and yet not notice this discontinuity.

Thus, the relative paucity of location familiarity and coherent ordering does not necessarily entail that dreams themselves are incoherent, but only that their coherence may be based on other qualities, such as emotion, narrative structure, or protagonist activity. Ambulatory motor activity, in particular, is prevalent in dream content (McCarley \& Hobson 1977) and sustains a sense of story continuity, even though the scenes through which movement occurs change frequently and unexpectedly. For example, one short dream (Hobson \& McCarley 1977) illustrating continuous motor activity also belies an absence of location continuity; in quick succession the dreamer reported: "sitting in front of a piano," "walking around an amusement park," "watching a band," "walking up some steps," "near rocks in the water," and "filling a hole up with marble slabs" (p. 117). This array seems too disparate and incoherent to support MoL-like memory enhancement.

It could be argued that since even fictitious MoL locations are mnemonically effective (Yates 1966), so too are the novel, fictitious locations of dreams. In fact, fictitious "virtual environments" are as effective for improving memory as is a standard, familiar MoL setting (Legge et al. 2012). However, the latter finding was for stimuli that were encoded immediately after exposure to the virtual environment, when memory for the environment had not yet dissipated. No testing of longer-term consolidation was undertaken, and it is doubtful that it could have occurred if the virtual environments were not also committed to memory as required by the MoL. Dream settings, though perhaps similarly "virtual," are also not typically highly memorized.

It could also be argued that MoL familiarity and coherence requirements need not be based upon geographic or architectural locations to be effective. As Llewellyn's "lawsuit" example illustrates, a well-structured tableau containing very closely spaced locations may be effective; dream imagery may be much like this. Note, however, that such closely spaced MoL landmarks are less effective than are distantly spaced landmarks, such as one's route to work (Massen et al. 2009). Nonetheless, this argument implies that dream locations may be irrelevant to dreaming's memory function; the essential ingredient may be the formation of composite dream images.

In conclusion, research on the question of dreaming's MoL-like quality suggests that dreams do not fulfill certain basic requirements; locations are neither familiar nor coherently ordered and thus may not facilitate episodic memory. This conclusion dovetails with most, but not all (cf. Griessenberger et al. 2012; Rauchs et al. 2004) evidence that either sleep does not consolidate episodic memory (Aly \& Moscovitch 2010; Inostroza et al. 2013) or that episodic memory depends upon non-rapid eye movement (NREM), rather than REM, sleep (Daurat et al. 2007; Drosopoulos et al. 2005; Scullin 2012; van der Helm et al. 2011a).

\section{Dreams, mnemonics, and tuning for criticality}

\section{doi:10.1017/S0140525X13001404}

\author{
Barak A. Pearlmutter ${ }^{\mathrm{a}}$ and Conor J. Houghton ${ }^{\mathrm{b}}$ \\ ${ }^{a}$ Department of Computer Science and Hamilton Institute, National University \\ of Ireland Maynooth, Co. Kildare, Ireland; ${ }^{\mathrm{b}}$ Department of Computer Science, \\ Bristol University, Bristol BS8 1UB, United Kingdom. \\ barak@cs.nuim.ie conor.houghton@bristol.ac.uk \\ http://www.bcl.hamilton.ie/ barak/ \\ http://www.bristol.ac.uk/engineering/people/conor-j-houghton/index.html
}

Abstract: According to the tuning-for-criticality theory, the essential role of sleep is to protect the brain from super-critical behaviour. Here we 
argue that this protective role determines the content of dreams and any apparent relationship to the art of memory is secondary to this.

It is widely believed that memory consolidation is the purpose of sleep. However, as detailed in the target article, the description of this process that emerges from experiment is confusing and complex. Mindful that complex phenomenology is commonly the attribute of a secondary purpose, we proposed (Pearlmutter \& Houghton 2009) that sleep has a different primary purpose tuning for criticality - and that the link with memory consolidation is secondary. Memory consolidation may occur during sleep, it may even occur preferentially during sleep, but it is not the essential purpose of sleep.

The obvious goals of learning, rapid responses to stimuli, and prolonged retention of short-term memories are, from a network dynamics point of view, attributes of near-critical systems. Thus, it is likely that, during learning, neuronal circuits become increasingly critical and approach super-critical behaviour-behaviour that would involve runaway oscillations and constitute a pathological disruption of normal brain function. Thus, the optimisation of behaviour during learning requires a mechanism for preventing the brain from straying into a parameter region where it could be stimulated into pathological oscillations, and learning can occur only if there is a margin of safety around the current state of the brain.

In the tuning-for-criticality theory the purpose of sleep is to establish this margin of error. Thus, during sleep there is a synaptic plasticity regime which is different from the one which supports learning during wakefulness, and this works to move the brain away from criticality. In this way the sleep-work cycle tunes the brain so that it is close to criticality and optimised to respond to likely stimuli, but safe from the uncontrolled behaviour associated with super-criticality.

According to this proposal, the role of dreams is to stimulate the brain in a manner that mimics awake cognition as part of a search for self-reinforcing loops. Dreams are therefore a guess, based on recent and distant memories, of possible future stimuli. The target article describes the attributes of dreams. Dreams are emotionally salient and sometimes shocking or disgusting, they are associated with pontine-geniculate-occipital waves, they are narrative but the narrative is fragmentary, they contain incongruities, and the identities of people in them are often fused or indeterminate. Dreams are largely visual, with snatches of auditory sensation but almost no olfactory, gustatory, or tactile content. They are often characterised by the illusion of movement and spatial navigation. The target article points to this as a congruence between the form of the classic mnemonic and dreams, though it does not elaborate on what cognitive or biochemical mechanism related to this congruence would act to improve the efficacy of the mnemonic. We contend, however, that these are all attributes which dreams might be expected to have if they are a mechanism the brain uses to cast around for neuronal circuits in which potential stimuli could evoke runaway oscillations.

Pontine-geniculate-occipital waves arise only in wakefulness in response to unexpected events and are believed to focus attention, heightening responses. Emotionally salient, disgusting, and shocking dream content is likely to provoke stronger neuronal responses. Because the brain responds to temporally integrated stimuli, the exploratory simulated environment produced by dreams needs a narrative structure, but for this purpose, the narrative may be fragmented and discontinuous. The dream world need not be wholly specific; fused and indeterminate aspects of dream content seem unlikely to reduce its capacity to evoke responses indicative of potentially harmful self-reinforcing loops. The visual, auditory, and ambulatory character of dreams reflects the timescales and stimulus types most pertinent to everyday learning and common threats. In short, the nature of dream content is consistent with a tuning-for-criticality function of sleep. The pertinence of dream content means that similar content is likely to be memorable and this might explain the utility of this type of content in mnemonics.
Learning for behavioural optimisation is a key objective of cognition and content salient to this objective is likely to be both memorable and provocative. It may be this, rather than a direct functional link, that relates the art of memory and the nature of dreams. Alternatively, we might speculate that the random activity involved in dreaming first evolved to avoid criticality and that the availability of random narratives based partly on recent memories encouraged the development of learning strategies which make strong use of these narratives through a sort of hashing algorithm. In this light, we would view mnemonic consolidation as a particularly apt secondary purpose of sleep, in that it makes use not only of physical quiescence but also exploits the computational processes already present in tuning for criticality.

Mnemonic consolidation may avoid waste by utilizing the dream content that was generated, at some metabolic expense, to locate potentially super-critical neuronal circuits. However, we believe that there is no evidence to suggest that memory processes like consolidation and mnemonic association can occur only during sleep. Sleep is such an extreme and hazardous adaptation it seems unlikely it evolved to serve a function, like mnemonic association, that could also be performed while awake.

\section{From Freud to acetylcholine: Does the AAOM suffice to construct a dream?}

\author{
doi:10.1017/S0140525X13001416
}

\section{Helene Sophrin Porte \\ Psychology Department, Cornell University, Ithaca, NY 14853. hsp2@cornell.edu}

Abstract: Toward illuminating the structure of Llewellyn's dream theory, I compare it in formal terms to Freud's dream theory. An alternative to both of these dream machines, grounded in the distribution of cholinergic activation in the central nervous system, is presented. It is suggested that neither "high" nor "low" dream theory is sufficient to account for the properties of dreams.

Llewellyn's is an attractive hypothesis, engagingly presented. In structure - not, of course, in substance - it is like Freud's dream theory. Freud's dream machine exercises a set of operations the components of the dream work - that, when applied unconsciously in waking, produce a neurotic symptom. Llewellyn's dream machine exercises a set of operations - the components of the ancient art of memory (AAOM) - that, when applied deliberately in waking, elaboratively encode an episodic memory.

Both dream generators account ingeniously for the peculiarities of dream experience, and both achieve an explicit aim. By condensation, displacement, "consideration of representability," and secondary revision, a Freudian dream expresses a forbidden Oedipal wish (Freud 1900/1981). A Llewellynian dream - by association, organization, narration, embodiment, movement, and location encodes the to-be-remembered material. In both cases the latent dream is obscured-in Freud's model by cunning, in order to evade the "psychic censorship," and in Llewellyn's (she has no use for a calculating homunculus) by guileless neural processes, in order to realize what I shall call the AAOM-in-REM. These formal likenesses entail another. Against the school of "bottom-up" dream construction, Llewellyn's theory, like Freud's, is a "high" theory: an explicitly "top-down" machine.

What if - both on the neural axis and in regard to imaginative complexity-dreaming is neither a "high" nor a "low" process, but an egalitarian one? A case in point is what might be called Llewellyn's telencephalocentric treatment of levels of acetylcholine (ACh) in waking and in sleep. Table 1 lists and describes the main cholinergic neural groups in the central nervous system. It is fair to ask, can the AAOM-in-REM accommodate the realities of ACh-in-REM, from hindbrain to forebrain? (In Table 1, cell 